\title{
Communicative Criteria for Usability Evaluation
}

\section{- experiences from analysing an e-service}

\author{
Stefan Cronholm \\ University of Borås \\ Linköping University \\ stefan.cronholm@hb.se
}

\begin{abstract}
Today we are primarily using computers for communication. We communicate via computers as professionals and at our spare time. One growing context of computer use is when we as citizens communicating with authorities. This paper suggests communication criteria for evaluation of public e- services. The suggested criteria are derived from a communication perspective and applied in a case study for evaluation of an e-service. Communication between authorities and citizens are often communication intensive and consequently conditions and consequences of communication should be evaluated. The aim of the suggested communication criteria is to be a complement to established traditional usability criteria.
\end{abstract}

\section{Author Keywords \\ Usability criteria, e-services, design principles, guidelines.}

\section{ACM Classification Keywords}

H.5.2. User interfaces (e.g. Evaluation methodology, Theory and methods).

\section{INTRODUCTION}

This paper is about evaluation of e-services. E-services are used for communication both at work and at home. At work, we communicate in order to do business. At home, we use e-services for communication with friends (e.g. facebook), with companies (e.g. booking tickets for travels or theatres, buying goods) or with governments (e.g. child care, tax inquiries). In all these cases, eservices are used as instruments for communication. In this paper we have studied an e-service that supports applications for construction of wind power plants.

An e-service is a type of IT-system. According to Seddon (2001), there is a huge amount of money spent on ITinvestments all over the world. Therefore, it is important to evaluate the outcome of the investment. Evaluation is never an easy task and consequently there are a lot of suggestions for how to conduct evaluations. A majority of the literature discussing evaluation takes a formal-rational view and sees evaluation as a largely quantitative process of calculating the likely cost/benefit on the basis of defined criteria (Walsham, 1993). There are also

OZCHI 2010, November 22-26, 2010, Brisbane, Australia. Copyright the author(s) and CHISIG

Additional copies are available at the ACM Digital Library (http://portal.acm.org/dl.cfm) or ordered from the CHISIG secretary (secretary@chisig.org)

OZCHI 2010 Proceedings ISBN: x-xxxxx-xxx-x interpretative approaches (e.g. Walsham, 1993, Remenyi et al., 1999) These interpretative approaches view ITsystems often as social systems that have information technology embedded into it Goldkuhl \& Lyytinen (1982).

To evaluate often includes using a set of specific measures or qualitative criteria. There are measures that are of harder technical or economic character and there are measures that are of softer user-oriented character such as user experience (Hassenzahl, 2008; Law et al. 2009). To reflect upon the choice of evaluation criteria is therefore important and cannot be seen as a routine-like task.

There are a lot of criteria, heuristics, guidelines or principles for designing or evaluating of IT-systems (e.g. Nielsen, 1994; Häkkilä \& Mäntyjärvi, 2006; Kärkkäinen \& Laarni, 2002; Dix et al., 2004; Muller et al., 1998; Shneiderman, 1998; Preece et al., 2007). Despite all these criteria, there are still many ill-designed IT-systems (Park, 2009). Thus, a reasonable question to ask is "are existing criteria not usable enough?".

In the literature, criticism of existing criteria can be found: Vredenburg et al. (2002) point out that there is a major discrepancy between the commonly cited guidelines and the actually applied ones, the work by Tao (2008) has identified a significant gap between the knowledge of design and application of web design guidelines, Chevalier \& Ivory (2003) claim there is little support for the complexities involved in the design activity, Gould \& Lewis (1985) have identified that design guidelines are limited since their descriptions are not detailed enough, and Burmester and Machate (2003) claim the designer has to understand the rationale behind the guidelines before applying them. Van Welie et al. (1999) discuss guidelines and heuristics as means for assistance in improving the usability. They claim that in practice available checklists, tests, guidelines etc. differ in terms of structure, content and terminology. Finally, Aitta et al. (2008) and Chisnell et al. (2006) claim that most heuristics are too broad or too general

The aim of this paper is to suggest communication criteria for e-services based on a communication perspective. Of course, there are several studies proposing guidelines for e-services (e.g. Hendersson et al, 2003; Nielsen, 1999). Guidelines suggested from these studies are based on other perspectives. 
The character of the relation between authorities and citizens is many times communication intensive. There are messages sent forth and back between the citizen and the authority. The communicative perspective in this study is thus motivated since the primary aim between a citizen and an authority is to reach their goals through communication. The communication perspective is described in section 2 . In section 3 , the case is briefly described and in section 4 the research approach is presented. In section 5, the findings are presented and finally in section 6 we present the conclusions.

\section{THEORETICAL BASIS}

An e-service is an umbrella term for services on the Internet (The Free Dictionary, 2009). One definition reads: "E-services include e-commerce transaction services for handling online orders, application hosting by application service providers (ASPs) and any processing capability that is obtainable on the Web". This definition is clearly derived from a business-consumer perspective. An e-service could also support relations between an authority and a citizen or a relation between an authority and companies. According to Burell (2006), a broader definition characterizes an e-service as: being available $24 / 7$, independent of geographical location, different from paper-based routines and implies a certain degree of selfservice.

In order to suggest and to validate criteria for e-services, we have applied a communicative perspective. The theoretical sources for viewing e-services as communication systems are: language action theory (e.g. Austin, 1962; Searle, 1969; Goldkuhl \& Lyytinen, 1982), theory about communication (e.g. Habermas, 1985; Winograd \& Flores, 1986) and social action theory (e.g. Dietz, 2001; Goldkuhl, 2009).

Followers of language action theory view communication as one type of action. One of the main messages in language action theory is that to communicate means to establish interpersonal relationships between a sender and a receiver (Searle, 1969). Theories about communication emphasizes, among other things, that communication is intentional. That is, messages contain more than a propositional content; they also have an intention. In speech act theory this is called the illocutionary force (Searle, 1969). The intentions in messages can be of different character. Some messages are requests of something while others might order you to do something. There are also messages that are sent purely for information purposes. We believe that the presence of the intention of the message should always be preserved. Furthermore, we believe this is especially important when communicating through an e-service since there can be a distance between the communicators.

Using a social action perspective means that it is not acceptable to view IT-systems as a black box with some social and organizational consequences (Dietz, 2001). According to Goldkuhl (2009) IT-systems "cannot be seen as repositories of facts of the world". The IT-system is a communicative instrument in the organisation. The employees in an organisation use the IT-system for conducting business actions. Thus, the IT-system mediates the communication sent between the employees. The communicative perspective emphasizes a user communicating with other users via a user interface (Goldkuhl, 2009; Röstlinger \& Cronholm, 2009).

This communicative view of IT-systems is also valid for e-services. The role of an e-service is to mediate communication between the citizens and the authority. To apply a communication perspective is to view the eservice as a medium for communication. It is a human-tohuman communication; not only human-computer interaction.

As pictured in figure 1, we have distinguished three types of qualities that should be supported by the e-service: interaction quality, communication quality and goal quality. Interaction is something that takes place between a user and the e-service. The communication takes place between two users (an authority administrator and a citizen (or a representative for a company). The communication is mediated by the e-service. The communication process is supported by the interaction quality (Röstlinger \& Cronholm, 2009).

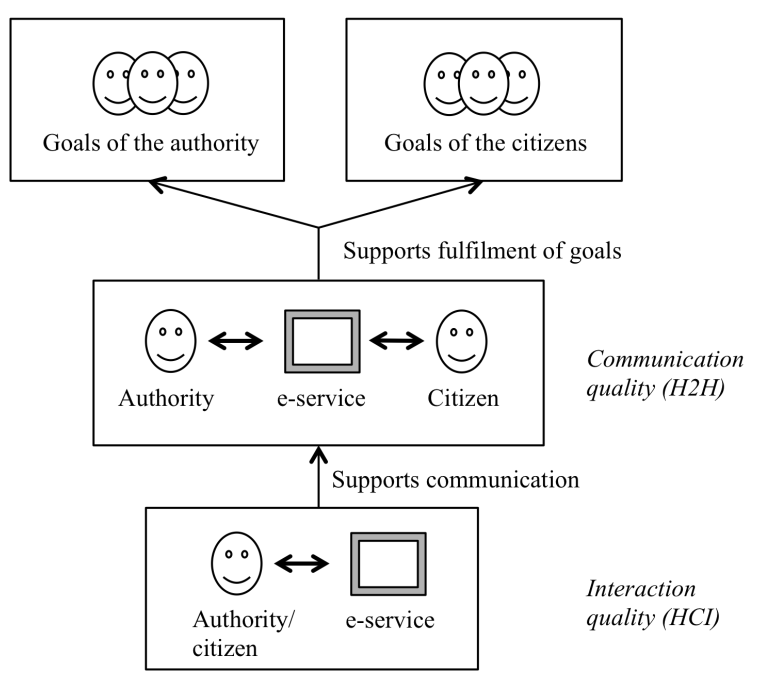

Figure 1. The relationship between interaction, communication and goals.

Finally, the communication quality supports the fulfilment of the goals of the authority and the goals of the citizens. Our understanding is that many public eservices primarily have been developed to support the goals of the authorities. That is, their aim is to simplify the work performed by the administrators of the authority. Of course, omitting the perspective of the citizens can cause negative attitudes. Thus, we have emphasized both the goals of the authority and the goals of the citizens. The relationship between goals, communication and interaction can be viewed as a goalmeans hierarchy.

As mentioned in section 1, there are several suggestions for evaluation criteria (e.g. Nielsen, 1994; Häkkilä \& Mäntyjärvi, 2006; Kärkkäinen \& Laarni, 2002; Muller et al., 1998; Shneiderman, 1998). According to our knowledge, most of these are supporting humancomputer interaction. We are not saying that these criteria are unusable; on the contrary they seem to provide good 
support for analyzing human-computer interaction, but they are not primarily supporting evaluation of e-service communication between humans.

\section{BRIEF DESCRIPTION OF THE CASE}

The analysed e-service is a result of three-year development project. The aim of the e-service is to support applications for construction of wind power plants. This e-service is called the wind service. The authority that issues permission for construction of wind power plants is the Swedish Energy Agency.

The e-service analyzed in this study concerns two relations: 1) between the authority and citizens, 2) between the authority and companies. There are companies specialized in constructing wind power plants and there are private citizens who want to build wind power plants on there own properties. Both companies and private citizens are interested in support for how to apply and to get permission from the authority in order to construct wind power plants.

The idea is that the e- service should contribute to a more effective handling of applications. The goals of the authority read: it should be easy for applicants to access necessary information and necessary support in order to decide where it could be appropriate to construct wind power plants in order to: 1) simplify the communication between authorities and applicants, 2) to shorten the time for handling applications and 3) offer the same information to all applicants and thereby secure that applicants have equal conditions.

Goals identified from interviews with applicants (citizens or companies) reads: 1) The information about conditions for constructing wind power plants must be updated, 2) the e-service should complement, not replace, the face-toface meeting with administrators, 3) as much information as possible regarding conditions for construction should be provided, 4) the e-service should be easy to use and 5) clear information about the application process.

Requirement specifications, design specifications and realization was developed by a group consisting of representatives from the Swedish Energy Agency, The National Board of Housing, Building and Planning, project consultants, IT-consultants and researchers. The development of the e-service started in the year 2006 and lasted three years. The development was financed by the Swedish Governmental Agency for Innovation Systems.

The main functionality of the wind service consists of a digital map where the applicants can get information relevant for analyzing where it is appropriate to construct a wind power plant. The applicants can add different layers on the map that for example visualize: where existing plants are located, where it is windy, where environment protected areas are located, where there are power supports and where there are seaways. The wind service is part of a larger e-service called the planning portal. The wind service will be launched late 2010 .

\section{RESEARCH APPROACH}

The aim of this study is to make a scientific contribution as well as a practice contribution. The scientific contribution consists of communicative evaluation criteria for public e-services. The contribution to the practice consists of evaluation results and recommendations for a re-design of the wind service. The processes of making a scientific contribution together with a practice contribution have been tight coupled (see figure 2).

The aim of the evaluation process viewed from a scientific perspective has been to identify and validate the suggested communication criteria. In this respect, the evaluation process can be seen as an empirical data source. Ideas for suggestions of criteria have also been deduced from theory (see section Theoretical basis) and from of other scholars (e.g. Goldkuhl, 2009, Ågerfalk, 2003, Cronholm \& Goldkuhl, 2002). Input from theory and empirical experiences from the evaluation have worked as a base for reflection and successive refinement. While we have evaluated the e-service we have reflected upon the criteria suggested by asking questions as: "How did this criterion support the evaluation?", "What phenomenon is analysed by using this criterion?" and "How did this criterion govern the analysis?" and "Is this criteria supporting interaction or communication?".

From a practice perspective the contributions consist of a criticism of the wind service and of recommendations for measures to take. The wind service has been evaluated with both a criteria-based approach and an open-minded approach. The difference between these approaches is that the criteria-based approach governs the attention of the evaluator. Using pre-defined criteria means to set a focus on something that is perceived as important. The open-minded approach means the opposite. To be openminded means to gather data on a broad array of actual effects and evaluating the importance of these effects in meeting demonstrated needs (Patton, 1990). This openminded approach is inspired by a strategy called "goalfree evaluation" (Patton, 1990). The aim of goal-free evaluation and to be more open-minded is to gain a deeper understanding of the nature of what is to be evaluated (Hirschheim \& Smithson, 1988).

The wind service was evaluated with both communication and interaction criteria. The aim of this paper is not to suggest interaction criteria. Therefore we are not presenting reflections of our usage of interaction criteria. The aim is to present communication criteria. The interactive criteria we have used are the Ten Heuristics (Nielsen, 1994) and Eight golden Rules (Shneiderman, 1998). The Ten Heuristics consist of: Visibility of system status, Match between system and the real world, User control and freedom, Consistency and standards, Error prevention, Recognition rather than recall, Flexibility and efficiency of use, Aesthetic and minimalist design, Help users recognize, diagnose, and recover from errors, and Help and documentation. The Eight Golden Rules are: Strive for consistency, Enable frequent users to use shortcuts, Offer informative feedback, Design dialog to yield closure, Offer simple error handling, Permit easy reversal of actions, Support internal locus of control, and Reduce short-term memory load. These interaction criteria can be viewed as means for the communication criteria (see section "Findings"). In the same way, the 
communication criteria should contribute to the fulfilment of the goals listed in the section "Brief Description of the Case".

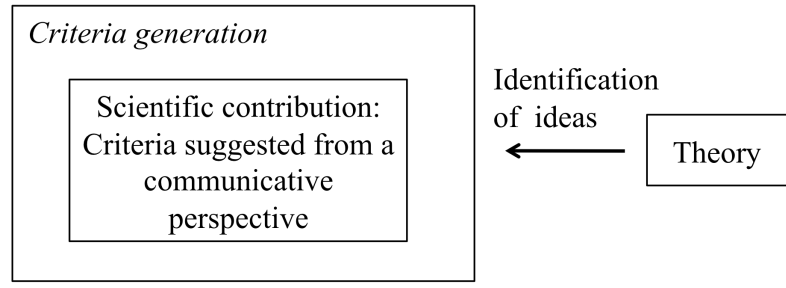

Testing $\downarrow \uparrow$ Identification, reflection, validation

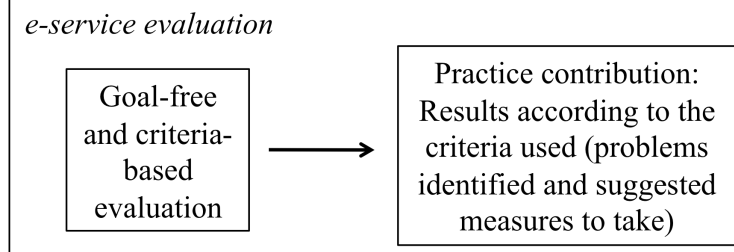

Figure 2. Research Approach.

\section{FINDINGS}

This section consists of two parts. In the first part we present the communication criteria. In the second part, we elaborate on the relations of the communication criteria to both the interaction criteria (see section "Research Approach") and to the goals (see section "Brief Discussion of the Case").

\section{Communication criteria}

There are nine suggested communication criteria. The criteria are: Actor clarity, Intention clarity, Satisfying communication needs, Clear action repertoire, Action transparency, Support for reading and formulation, Utilization of the medium potential, Integration of contextual information integration, Categorize information and use menu items that are unequivocal.

\section{Actor clarity}

Criteria description: This criterion is concerned with the possibilities to identify the sender of a message. Who is communicating? Information in IT-systems' databases have often, unfortunately, a tendency to "objectify" messages and strip away the senders from the messages. What is kept is often only the message content and not information about message origin (i.e. the sender). To know about the senders (i.e. the originators) of different messages is a way for users to be better informed about what is conveyed. There may arise needs for getting more information which leads to a need for the user to get into contact with the sender; either through the system or besides the system. To have visible senders means more transparent systems. This criterion is a call against anonymity.

Evaluation of the wind service: There is no doubt who the communicator is. There is always a name and a role of the person responsible for the information presented. For example, if the applicant wants detailed information about where environment protected areas are located, there is clear contact information to the responsible information provider.

\section{Intention clarity}

Criteria description: This criterion is concerned with the precision and communication clarity of messages. It states that messages should not only contain a propositional content, but also a clear intention; or stated in terms of speech act theory a clear illocutionary force (Searle, 1969). Many times there can be an uncertainty about the meaning of messages. Is the message sent to inform me about something or am I supposed act upon the content in the message? Is the message an order or a request? Doubts of this kind should be eliminated.

Evaluation of the wind service: The intentions of messages are clearly stated. The applicant is informed about which actions he is suppose to take. An example is that before an application is approved or not, other stake holders (such as neighbours) that are affected by the decision have to be informed about the application in order to say their meaning. This is an example when a message is more than pure information; the message is also an encouragement for action.

\section{Satisfying communication needs}

Criteria description: This criterion is concerned with the possibility to fulfil the users' communication need (both the citizens and the authority's needs). Are there possibilities for the user to formulate desired messages? That is, can the user say what he or she wants to say through the system or must other communication channels be used? Is it possible, not only to express routinized messages, but also to express the exceptional and unexpected? The criterion is concerned with the semantic expression capability of the system.

Evaluation of the wind service: There are both satisfying and unsatisfying communication needs in the wind service. An example of a satisfying communication need is that the user can get information about where it is appropriate to construct wind power plants. An example of a unsatisfying communication need is that the user can't apply for the construction through the wind service. A paper-based application has to be sent outside the wind service.

\section{Clear action repertoire}

Criteria description: The system affords a repertoire of possible actions (functionality) to the user. The sum of these actions can be viewed as the e-service's communication ability. In order to perform any actions, the user needs to identify and understand what the action potential is. The former criterion, "satisfying communication needs" is concerned about what can be communicated. This criterion is concerned about how well the action repertoire is presented. The e-service should support the users' understanding about what actions can be conducted and where in the system they can be accessible.

Evaluation of the wind service: On the top level the wind service offers five menu items: Open the map, Information about wind power, Effects on the animal life, Effects on the environment and, The application process. These menu items reflect the action repertoire. Some of the menu items could be labelled more clearly (see 
criterion Use menu items that are unequivocal). The subsequent web pages are informative; especially the page about wind power information. This page informs why you should use wind power and provides statistics of the performances of existing power plants. The menu item "Open the map" offers access to a map where the user can analyse information in order to decide the best location for constructing a wind power plant. How to use the actions offered are easy to understand.

\section{Action transparency}

Criteria description: This criterion is concerned with the possibility to understand the meaning of an action beforehand. What will happen after the user has conducted an action such as pressing a certain button? What are the communication effects? The criterion states that the user should understand the communication effects in advance and that the actions offered should be transparent.

Evaluation of the wind service: Through the e-service the transparency is clear. It is easy, and in advance, to understand what will happen when a menu item is selected or button is pressed.

\section{Support for reading and formulation}

Criteria description: Many authorities still offer e-services that only entails reading the documents. That is, the citizen can read documents provided by the authority. This one-way communication does not support formulation of messages from the citizen to the authority (these e-services are immature and maybe they should not be called e-services at all). A consequence of only permitting reading-functionality is that the communication between the citizen and the authority has to use other communication channels in parallel such as telephone, e-mail and face-to-face meetings. A more developed e-service supports both reading and formulation of messages (Goldkuhl \& Persson, 2006).

Evaluation of the wind service: The wind service can be seen as a hybrid. Parts of the wind service are highly interactive such as the map, while other parts are for reading such as the information about the wind power. This part informs the user of why wind power should be used with statistics attached. The statistics are very exhaustive and presented on numerous lists; but the information is only for reading (pdf). There is no designed way for the user to formulate questions based on the statistics. There is no way for the user to filter or to sort the information.

\section{Utilization of the medium potential}

Criteria description: An e-service is an electronic medium. That is, the e-service has another potential in supporting users compared to a paper-based procedure. Unfortunately, there are many e-services that haven't utilized this potential and paper-based procedures are sometimes copied unreflected into the new electronic media. The e-service is a computer-based tool that can be used for 1) simply tasks and 2) make tasks possible to conduct. To simplify tasks means that tasks can be performed faster with less effort. To make tasks possible to conduct means that tasks that were not possible to conduct in a paper-based procedure are now with support from the e-service possible to perform.

Evaluation of the wind service: Unfortunately, the wind service has not utilized the medium potential. For example, the communication quality has not been supported by the use of electronic forms (applications). There are no automatic controls that check if the form contains required information. A common complaint from administrators is that forms are incomplete or not readable. Another example is that the information provided is not based on the user's previous actions. Much of the information provided is not relevant. The information could be a selection and it could be sorted based on the user's preferences (e.g. sorting, filtering, aggregating). Furthermore, the user could be guided through the application process. All these examples can be viewed as a means for improving the communication quality. It seems like a proper analysis of the application process is missing and that the question of how the eservice could support both the administrators and the applicants never have been asked.

\section{Integration of contextual information}

Criteria description: To communicate with an authority often means that the user needs information from several units within the authority. It can also mean that different authorities are involved. Public e-services are often interlinked to each other. A citizen that needs information located in more than one e-service is often forced to follow these links.

Unfortunately, many times these links lead to the top level in the next e-service. This means that the citizen is forced to leave the context, that is, the ongoing activity or work task the user is involved in. From this top level in the next e-service the user has to interpret the action repertoire in order to navigate in the information structure to find the information needed. The user can perceive this navigation hard since many e-services can be regarded as information intensive; especially if they contain regulations or other formal authority information.

One reason for offering an e-service is often that authorities want to be able to handle errands more efficiently and to reduce unnecessary communication with citizens. Thus, it is important that the e-service supports the user to be as informed as possible and that it is easy to get the information needed. Being informed implies good conditions for action and communication.

The description above is an example of bad design. This is an example of when it has been considered to be more important to simplify the designers/programmers work instead of the citizens. Instead, contextual information should be integrated and easily accessible. The user should not be forced to leave the context of the task.

Evaluation of the wind service: There are several links to other organizations that can provide information about wind power plants and the application process. Unfortunately, the designers/programmers of the wind service have not prioritized contextual information integration. There are links leading to top pages in other e-services and there are also many broken links. In this 
respect, the conditions for communication support are considered as weak.

\section{Categorise information and use menu items that are unequivocal.}

Criteria description: The information presented should be categorized. That is, similar information should be grouped and presented in the same menu group. Furthermore, items in a menu group should be unequivocal. Users should not have to guess what a menu item means. To use menu items that are unequivocal means to label menu items in an informative way and as mutually exclusive as possible. A good idea can be to label a menu item with action (verb) and an object (noun). The combination of these two word classes will give the user a pretty good idea to the meaning of the menu item.

Evaluation of the wind service: In general, the information presented is categorized in an understandable way. However, there is one exception. The user can add different layers on the map in order to get a good idea of where to build the wind power plant (e.g. power support, environmental protection, how much is blows etc.). These layers are categorized in a somewhat confusing way. The labels of the layers are: "Conditions for wind power", "Location for existing wind power plants", "Environmental protection", "Cultural protection" and "National interests". Examples of a confusing categorization are: 1) the first category is labelled "Conditions for wind power". A reasonable question to ask is "Are not all the layers conditions for wind power?" and 2) one of the members in the category "Conditions for wind power" is labelled "National interests of wind power". Why is this layer not a member of the category "National interests"

Furthermore there are two menu items called "Effects on the animal life" and "Effects on the environment". They are not mutually exclusive since animals are part of the environment. Furthermore, the menu item "Effects on the animal life" refers to a web page where protection of bats and birds are described. For some non-transparent reason the designer of the wind service has decided to bring forward these animals while protection of animals like rain deer and seal are described on lower levels in the information structure.

\section{Relations between goals, communication criteria and interaction criteria.}

In the section "Research Approach", the relations between interaction, communication and goals were discussed in terms of a goal-means hierarchy (see figure 1). A closer look at the interaction criteria reveals that they are primary supporting evaluation of humancomputer interaction while the communication criteria are primary formulated as a support for human-to-human communication.

Examples of typical interaction criteria are: "Visibility of system status", "Enable frequent users to use shortcuts", and "Permit easy reversal of actions". Examples of typical communication criteria are: "Actor clarity", "Intention clarity", and "Satisfying communication needs". However, some criteria can be characterised both as an interaction criterion and as a communication criterion. The suggested communication criterion "Categorize information and use menu items that are unequivocal" can be perceived as an interaction criterion since it is addressing the way menu items should be categorized. At the same time, the criterion supports communication since the information that should be categorised can be seen as a message sent from the authority. The authority is communicating something to the applicant (the user).

Some of the interaction criteria reside on higher abstraction levels. One example is "Match between system and the real world". The description of the criterion reads: The system should speak the users' language, with words, phrases and concepts familiar to the user, rather than system-oriented terms. This description is oriented towards conditions for communication.

The goals described above (see section Brief Description of the Case) are primary formulated as high-level business goals. Examples of goals of the authority are: "Simplify the communication between authorities and applicants" and "Shorten the time for handling applications". Examples of goals of the applicants are: "The e-service should complement, not replace, the faceto-face meeting with administrators" and "As much information as possible regarding conditions for construction should be provided".

One result from this brief analysis is that there is a difference in how the interaction criteria, the communication criteria and the goals are formulated. They set a focus on different aspects of the application process and they are formulated on different abstraction levels. The advantage and role of a multilevel abstraction hierarchy is discussed in Rasmussen et al. (1994). Rasmussen et al. compares a multilevel abstraction hierarchy with a means-end hierarchy and claim that a multilevel abstraction hierarchy is often used in practical problem solving processes.

The model presented in Figure 1 is an abstraction hierarchy consisting of goals and means. This hierarchy can be justified by several examples. One example is that the goal "Shorten the time for handling applications" is supported by many means such as: "Actor clarity", "Intention clarity" and "Satisfying communication needs", "Integration of contextual information integration", and "Categorize information and use menu items that are unequivocal". The communication criteria "Integration of contextual information" is supported by many interaction criteria such as: "Design dialog to yield closure" and "Reduce short-term memory load".

\section{CONCLUSIONS}

The scientific contribution of this paper is a number of communication criteria and a theoretical model describing the relations between interaction, communication and goals (see figure 1). There are a lot of general suggestions for usability criteria, design principles, heuristics or guidelines (e.g. Dix et al., 2004; Shneiderman, 1998; Gelderblom, 2008; Mayhew, 1992; 
Nielsen, 1994; Preece et al., 2007). According to our understanding they are primarily focusing on the humancomputer interaction aspect. There are also several suggestions concerning specific guidelines for e-services. Shneiderman (2000) suggests a number of guidelines aiming at elicit trust by users. According to our understanding, these guidelines are generated from a security perspective and from a juridical perspective. From this point of view these guidelines can be characterised as conditions for communication. Another study suggesting guidelines for e-services is performed by Santos (2003). These guidelines seem promising and relates to the human-computer interaction level.

We believe that our findings can be seen as complement to the existing suggestions. Existing interaction criteria provides a good support for analysing human-computer interaction, but they are not focusing human-to-human communication. The aim of the suggested criteria is to provide good conditions for communication between a citizen and an authority. Communication consists of both formulating messages and reading messages. The suggested criteria support both formulation and reading. Examples of criteria that support formulation are "Satisfying communication needs", "Clear action repertoire", "Action transparency" and "Utilization of the medium potential". Examples of criteria that support reading are "Intention clarity", "Integration of contextual information integration" and "Categorize information and use menu items that are unequivocal". Some of the communicative criteria support both formulation and reading.

The aim of the theoretical model is to encourage the evaluator to analyse e-services from different abstraction levels (interaction, communication and goals). The model has in this study been useful. However, a more precise analysis of the relations between specific goals, communication criteria and interaction criteria is needed.

The practice contribution consists of evaluation results of the wind service. The evaluation has revealed both strengths and weaknesses. The strengths and weaknesses revealed have been a base for recommending measures to take. The communication criteria can thus, in this case study, be considered as valuable. According to Yin (2003), "case studies, like experiments, are generalizable to theoretical positions and not to populations or universes. In this sense, the case study, does not represent a sample, and in doing a case study, your goal will be to expand and generalize theories (analytical generalization) and not to enumerate frequencies (statistical generalization)". That is, the researcher should generalise beyond the theory's empirical base.

We believe that the communication criteria are valid for other domains with similar conditions. We can think of two other domains. One domain concerns other contexts where there is an e-service supported communication between authorities and citizens (e.g. child care, tax inquiries). We think that most (if not all) criteria can be valid for this type of context. Another domain concerns a context where a customer is communicating with a supplier via an e-service (e.g. buying goods). We believe that several of the suggested communication criteria can inspire design or evaluation of commercial e-services.

\section{REFERENCES}

Aitta M-R., Kaleva S., Kortelainen T., Heuristic evaluation applied to library web services, New Library World, Vol 109, Nr 1/2, pp 25-45 (2008)

Austin, J.L., How to do things with words, Oxford University press. (1962)

Burell, M., Personas - a way to represent users with special needs in design of public e-services. Student thesis. Department of computer and Information Science, Linköping University. (2006)

Burmester, M., Machate J., Creative Design of Interactive Products and Use of Usability Guidelines - a Contradiction. In: Human-Computer Interaction: theory and practice (eds Julie A. Jacko, Constantine Stephanidis, Don Harris). Lawrence Erlbaum Associates. (2003)

Chevalier, A., Ivory, M.Y., Web site designs: Influences of designer's expertise and design constraints. In: International Journal of Human-Computer Studies, 58, 1, p. 57-87. (2003)

Chisnell, D.E., Redish, J.C.G., Lee, A. "New heuristics for understanding older adults as web users", Technical Communication, Vol. 53 No.1, pp 39-59. (2006)

Cronholm S. \& Goldkuhl G. Actable Information Systems - Quality Ideals Put Into Practice. In proceedings of the Eleventh Conference On Information Systems (ISD 2002). Sep 12-14, Riga, Latvia. (2002)

Dietz J L G., DEMO: Towards a discipline of organisation engineering. In: European Journal of Operational Research (128:2) pp 351-363. (2001)

Dix, A., Finlay, J., Abowd, G., D and Beale, R. HumanComputer Interaction. Pearson Education Ltd, Harlow, (2004)

Gelderblom, J.H., Designing Technology for Young Children: Guidelines Grounded in a Literature Investigation on Child Development and Children's Technology, in School of Computing, (2008)

Goldkuhl, G., Pragmatic Qualities of Information Systems - Actability Criteria for Design and Evaluation. In: the $11_{\text {th }}$ International Conference on Informatics and Semiotics in Organisations (ICISO), invited paper. Beijing, China (2009)

Goldkuhl, G., Lyytinen, K., A language action view of information systems. In: proceedings of the 3rd international conference on information systems, Ann Arbor, Michigan, pp. 13-31. (1982)

Goldkuhl, G; Persson, A; (2006) Characteristics of Public E-services: Investigating the E-diamond Model Proceedings of the First International Pragmatic Web Conference, Stuttgart

Gould, J.D., Lewis, C., Designing for usability: key principles and what designers think. In: Commun. ACM, 28,3, p. 300-311. (1985) 
Hassenzahl, M., User experience (UX): towards an experiential perspective on product quality. In Proc. of the 20th international Conference of the Association Francophone D'interaction Homme-Machine. IHM '08, vol. 339. (2008) ACM, New York, NY, 11-15. (2008)

Habermas, J.. The theory of communicative action. Volume 1. Reason and the rationalization of society. Beacon Press. (1985)

Henderson, J., McGoldrick, E., McAdam, R., A critical review of e-service in Northern Ireland Electricity. In Managing Service Quality, Volume: 13 Issue: 6 Page: 463 - 470. MCB UP Ltd (2003)

Hirschheim, R., Smithson, S., A Critical Analysis of Information Systems Evaluation. In: IS Assessment: Issues and Changes (eds $\mathrm{N}$ Bjorn-Andersen \& $\mathrm{G} \mathrm{B}$ Davis), North-Holland, Amsterdam. (1988)

Häkkilä, J., Mäntyjärvi, J., Developing design guidelines for context-aware mobile applications. In: ACM International Conference Proceeding Series; Proceedings of the 3rd international conference on Mobile technology, applications \& systems. Bangkok, Thailand, ACM. (2006)

Kärkkäinen, L., Laarni J., Designing for small display screens. In: ACM International Conference Proceeding Series; Proceedings of the second Nordic conference on Human- computer interaction. Aarhus, Denmark, ACM, p. 227-230. (2002)

Law, E., Roto, V., Hassenzahl, M., Vermeeren, A., and Kort, J., Understanding, Scoping and Defining User eXperience: A Survey Approach. Proc. CHI'09, ACM SIGCHI conference on Human Factors in Computing Systems. (2009).

Mayhew, D.J. Principles and Guidelines in Software User Interface Design. Prentice Hall, Inc, New Jersey, (1992)

Muller, M.J., Matheson, L., Page, C., Gallup, R., Methods \& Tools: participatory heuristic evaluation. In: Interactions, pp. 13-18. (1998)

Nielsen, J., Usability Engineering, Academic Press, Boston. (1993)

Nielsen, J., Heuristic Evaluation. In: Usability Inspection Methods (eds. Nielsen, J., Mack R. L.). John Wiley \& Sons. New York. (1994)

Nielsen, J., Designing Web Usability. (1999)

Park, J., Kim, J., Effects of contextual navigation aids on browsing diverse Web systems. In: Proceedings of the SIGCHI conference on Human factors in computing systems. (2009)

Rasmussen, J., Pejtersen, A.M., \& Goodstein L.P. Cognitive Systems Engineering, Wiley \& Sons Inc. (1994)

Röstlinger, A., Cronholm, S. Design Criteria for Public eServices. In: procedings of the 17th European Conference on Information Systems (ECIS). June 8-10, Verona, Italy. (2009)
Patton, M.Q., Qualitative Evaluation and Research Methods, second edition. Sage Publications. London. (1990)

Preece, J., Rogers, Y. and Sharp, H. Interaction Design: Beyond Human-Computer Interaction. John Wiley \& Sons Ltd, Chichester, (2007)

Remenyi, D., Sherwood-Smith M., Maximise Information Systems Value by Continuous Participative Evaluation". In: Logistics Information Management. Vol 12, No 1/2, pp 14-31. (1999)

Santos J., E-service quality: a model of virtual service quality dimensions, Managin Service Quality, Vol 13, Issue 3 (2003)

Searle, J.R., Speech Acts - an Essay in the Philosophy of Language. Cambridge University Press. (1969)

Seddon, P., IT Evaluation Revisited: Plus !a Change, In: proceedings of Eight European Conference on Information Technology (ECITE). Oxford, United Kingdom. (2001)

Shneiderman, B., Designing the user interface: Strategies for effective human-computer- interaction. 3rd ed, Addison Wesley Longman, Reading, Mass. (1998)

Shneiderman, B., Designing Trust into Online Experiences, Communications of the ACM, Vol 43, Issue 12, pp 57-59 (2000)

Tao, Y.-H., Information system professionals' knowledge and application gaps toward Web design guidelines. In: Computers in Human Behavior, 24,3, pp. 956-968. (2008)

The Free Dictionary, http://encyclopedia2.thefreedictionary.com/e-services (2009)

van Welie, M., Van Der Veer, G.C., Eliëns, A., Breaking down Usability. In: proceedings of Interact ' 99. Edinburgh, Scotland! 30th August - 3rd September. (1999)

Vredenburg, K., Mao J.Y., Smith P.W., Carey T., A survey of user-centered design practice. In: Proceedings of the SIGCHI conference on Human factors in computing systems: Changing our world, changing ourselves. Minneapolis, Minnesota, USA: ACM Press. (2002)

Walsham, G., Interpreting Information Systems in Organisations. Wiley \& Sons. (1993)

Winograd, T., Flores, F., Understanding computers and cognition: A new foundation for design Ablex, Norwood. (1986)

Yin, R.K., Case Study Research - design and methods, third edition, Oxford University Press, London. (2003)

Ågerfalk, P. J., Information Systems Actability: Understanding Information Technology as a Tool for Business Action and Communication, $\mathrm{PhD}$ diss, Department of Computer and Information Science, Linköping University. (2003) 